\title{
The gut microbiota and psychiatric illness
}

\author{
Glenda MacQueen, MD, PhD; Michael Surette, PhD; Paul Moayyedi, MB, PhD
}

\begin{abstract}
The global market for probiotics is projected to be worth almost \$USD 100 billion by 2020, ${ }^{1}$ reflecting growing acceptance that our intestinal microbiota can influence physiologic systems, including but not limited to the gut. Many lay publications enthusiastically tout the potential health benefits of an optimized microbiome - or conversely, the risks of dysbiosis. Depression, stress, anxiety and autism are all proposed to be at least partially sensitive to manipulation of the gut microbiome. Studies have suggested that a variety of conditions are influenced by the microbiome, including obesity, functional gastrointestinal (GI) disorders, chronic fatigue syndrome and inflammatory illnesses. All of these disorders also have an important central nervous system component. It is likely that a substantial portion of people who consume prebiotics or probiotics will do so with the aim of improving symptoms related to the brain.
\end{abstract}

\section{How strong is the evidence on which decisions to take prebiotic or probiotic preparations are being made?}

How strong is the evidence? In brief, not as strong as it needs to be. Animal studies consistently report that behaviour and the function of the nervous system are modulated by gut microbiota (see the reviews by Dinan and Cryan, ${ }^{2}$ Foster $^{3}$ and Anglin and colleagues ${ }^{4}$ ). Perturbing the gut microbiota in mice with antibiotics is associated with altered brain-derived neurotrophic factor in the hippocampus and amygdala and an increase in mouse exploratory behaviour, considered a surrogate for decreased anxiety. ${ }^{5}$ Chronic administration of Lactobacillus rhamnosus str. JB1 also increased exploratory behaviour, ${ }^{6}$ and this effect was associated with brain regionspecific changes in the $\gamma$-aminobutyric acid (GABA) system and was dependent on vagal integrity. ${ }^{6}$ Bifidobacterium longum NCC3001 normalized anxiety in mice with chemically induced low-grade inflammation. ${ }^{7}$ In contrast, other bacteria can induce anxiety-like behaviour in rodents, and this is effect also appears to be mediated by the vagus nerve. ${ }^{8,9}$ Social disruption in rodent models is associated with reduced richness and diversity of the gut microbial community, along with shifts at the level of operational taxonomic units (OTUs; groups of micro-organisms clustered by DNA sequence simi- larity), suggesting a bidirectional component to brain-gutmicrobiota relations. ${ }^{10,11}$ Diet may also play a role. ${ }^{12}$ Animal studies, therefore, provide strong support to the notion that altering the gut microbiota might result in altered behaviour.

Despite these persuasive animal data, relevant data from humans are extremely limited. Rather, the literature is mostly populated by reviews describing the potential of pre- and probiotics to treat psychiatric conditions. A search of PubMed in January 2017 yielded almost 400 results from a search limited to reviews of "brain microbiome." Searching for "depression microbiome" alone yielded more than 100 reviews, and "anxiety microbiome" and "autism microbiome" yielded slightly fewer.

In contrast, running the same searches requesting clinical trials rather than reviews will yield only a few articles, and even fewer are actually clinical trials relevant to the condition. The human clinical data supporting a role of pre- or probiotics in other diseases, such as GI conditions, is stronger, but still limited. Systematic reviews suggest that probiotics are effective for symptoms of irritable bowel syndrome, for example, although a particular probiotic could not be recommended because studies used various species and strains. ${ }^{13}$

Although the paucity of data confirming the ability of the microbiome to enhance nervous system function is striking, there are a few recent studies that have yielded preliminary data supporting the notion that the intestinal microbiota can influence human behaviour. Steenbergen and colleagues ${ }^{14}$ had 20 healthy participants consume either a multispecies probiotic or identical placebo for 4 weeks and found that the probiotic group had reduced cognitive reactivity to sad mood. These findings are consistent with those of a study in which healthy volunteers who took 1 of 2 prebiotics for 3 weeks had decreased attentional vigilance to negative versus positive information in a dot-probe task. ${ }^{15}$ In that study, however, there were more measures (anxiety, perceived stress, memory) that did not change with either of the prebiotic products, making it hard to evaluate the significance of change on the dot-probe only. ${ }^{15} \mathrm{~A}$ small study reported that women who consumed fermented milk product with probiotics had changes in midbrain connectivity during an emotional attention task compared with women who did not consume the product with probiotics. ${ }^{16}$

Correspondence to: G. MacQueen, Room 7D14, TRW Building, 3280 University Dr. NW, Calgary AB T2N 4Z6; gmmacque@ ucalgary.ca

DOI: 10.1503/jpn.170028 
There have been a small number of case-control studies comparing gut microbiota of patients with psychiatric disorders to healthy controls. For example, Jiang and colleagues ${ }^{17}$ noted that despite "profound interindividual variability," Bacteroidetes, Proteobacteria and Actinobacteria were increased in 46 people with active or treated major depressive disorder (MDD), whereas Firmicutes were reduced compared with 30 healthy controls. ${ }^{17}$ Another recent study noted alterations in the phyla Firmicutes, Actinobacteria and Bacteroidetes in patients with MDD. ${ }^{18}$ Whether alterations in the microbiome contributed to the onset of MDD or emerged as a result of the illness or pharmacological treatment is unknown. One recent study found that there was no significant difference in species richness between depressed and nondepressed participants, ${ }^{19}$ although another study reported that depression was associated with decreased gut microbiota richness and diversity ${ }^{20}$ and another reported lower Bifidobacterium counts in patients with MDD. ${ }^{21}$

Fecal microbiota transfer from patients with MDD to microbiota-deficient animals can induce behavioural changes in the recipient animal that are considered analogues of depression or anxiety. ${ }^{18,19}$

There are few reports of randomized controlled trials evaluating the effect of probiotics in psychiatric patients. Tomasik and colleagues $^{22}$ randomized 58 people with schizophrenia to 14 weeks of adjunctive Lactobacillus rhamnosus strain GG and Bifidobacterium animalis subsp. lactis strain Bb12 or placebo. Probiotic treatment reduced levels of immunomodulatory proteins, but the clinical implications are unclear as there were no psychiatric outcomes measured in the trial. In another study, 40 people with major depression received either a capsule containing Lactobacillus acidophilus, Lactobacillus casei and Bifidobacterium bifidum or placebo for 8 weeks. ${ }^{23}$ Those treated with the probiotic preparation had a significantly greater degree of improvement on the Beck Depression Inventory than those taking placebo.

There are clinical trials of probiotics for depression and/or anxiety in various stages of planning and running according to clinicaltrials.gov. However, many have small sample sizes and most have only 1 probiotic preparation. Given the large number of probiotics on the market containing single strains and combinations of organisms, the uncertainty as to which patient might benefit and what dose and what route to administer the probiotic, it is unlikely that these trials will provide clarity on the efficacy of probiotics in psychiatric disorders in the near future.

Furthermore, probiotics are just 1 approach to change the gut microbiome. There are even fewer data on diet, antibiotics and fecal microbiota transplant therapy, some or all of which may have a role in modulating the gut microbiome to improve psychiatric illness.

\section{What are the barriers to microbiome research in people with psychiatric illnesses?}

There are substantial challenges to elucidating the role of the microbiome in human health and disease. A basic challenge is that there is no definition of what constitutes a healthy gut microbiota. ${ }^{24}$

The term "dysbiosis" is widely used, but is a concept rather than a defined entity, and no researcher can confi- dently state that an individual has an "unhealthy" gut microbiome. There is large variability among individuals in the composition of the microbiome, superimposed on variation in genetic and epigenetic susceptibilities to disease and dietary and environmental exposures. Thousands of microbial species inhabit human populations, and there is no universal definition of a microbial "species" or OTUs across studies. The distributions of microbes across hosts vary greatly, providing challenges to statistical descriptions of human microbiome data. As noted above, there is little consensus in the literature regarding what species are most likely to have therapeutic properties, and it may be the case that a balance of organisms, supported by a specific diet, provides the most benefit for any particular health condition. Trials of single or a few species may not represent an adequate approach to manipulating an individual's microbiome.

Finally, describing alteration in the microbiota of people with psychiatric illness requires a multidisciplinary team, including disciplines such as psychiatry, neurobiology, informatics, microbiology, gastroenterology and epidemiology. Such a team would be difficult to assemble and coordinate.

\section{Are there other ways to approach the study of the brain and microbiome?}

Patients with GI disorders have higher rates of anxiety and depression than the general population ${ }^{25}$ and those with other chronic diseases. ${ }^{26}$ This applies to both patients with inflammatory bowel disease (IBD) ${ }^{27}$ and those with irritable bowel syndrome (IBS). ${ }^{28}$

The corollary is also true; patients with anxiety and depression have more GI symptoms than healthy controls. ${ }^{29}$ A group recently reported that fecal microbiota signatures are similar between patients with depression and those with IBS in that they were less diverse than samples from controls and had similar abundances of alterations. ${ }^{30}$ Although the gut-brain axis has been widely recognized, the nature of these interactions remains poorly understood. Studying psychiatric symptoms in people with and without GI disease may provide further insight into associations between diet, the microbiome, intestinal physiology and the neurobiology underlying some psychiatric illnesses.

A recent Canadian Institute for Health Research (CIHR)funded Strategy for Patient-Oriented Research (SPOR) chronic disease network aims to explore this further. The Inflammation, Microbiome, and Alimentation: Gastro-Intestinal and Neuropsychiatric Effects (IMAGINE) network will assess diet, microbiome and a host of biological variables in 8000 participants with IBD and/or IBS as well as controls with no GI symptoms or disease. It is expected that approximately $20 \%$ of the cohort will have depression and/or anxiety, and this will be captured by including investigation of symptoms of depression and anxiety in a large cohort of patients with and without GI disease. The study is supported by a CIHR SPOR grant and is also funded by many partners and aims to follow participants for up to 4 years. A key hypothesis of the research program is that alterations in the gut microbiome may also drive anxiety and depression associated with these GI disorders. A portion of the cohort will participate in treatment trials that 
will allow for testing of novel therapies for the treatment of IBD and IBS and the psychiatric disorders associated with these diseases. The research network supporting this program is multidisciplinary and engages gastroenterology, microbiology, epidemiology, informatics psychiatry and psychology, encompassing both adult and pediatric disease. In the next few years, the IMAGINE network may be positioned to provide some of the most robust information available on the role of diet and the microbiome not only in individuals with GI disease, but also in those with depression and anxiety.

There is interest among both the research and lay communities in understanding the effects of the microbiome on the brain. Patients and clinicians alike are keen to understand whether modifying the microbiome might provide a treatment avenue for various neuropsychiatric conditions. Despite a relative abundance of reviews of the microbiome in human mental health and disease, actual data are sparse, and the widespread use of probiotics is not currently supported by randomized controlled trial data. Research programs that are comprehensive and bring together investigators from various disciplines may provide the best opportunity to move this exciting but challenging field forward in the next decade.

Affiliations: From the Department of Psychiatry, Mathison Centre for Mental Health Research and Education, Hotchkiss Brain Institute, University of Calgary, Calgary, Alta. (MacQueen); the Department of Biochemistry and Biomedical Sciences and Department of Medicine (Gastroenterology), McMaster University, Hamilton, Ont. (Surette); and the Division of Gastroenterology and Audrey Campbell Chair in Ulcerative Colitis Research, Department of Medicine, McMaster University, Hamilton, Ont. (Moayyedi).

Funding: The IMAGINE network is funded by CIHR, McMaster University, University of Calgary, Crohn's Colitis Canada, Takeda Pharmaceuticals, CEGIIR University of Alberta, Alberta Innovates, Manitoba Research, Allergan Inc, Queen's University, Dalhousie University and the Montreal Heart Institute Research Centre.

Competing interests: G. MacQueen has received honoraria from Allergan, Pfizer, Lundbeck and Janssen. P. Moayyedi has accepted speaker fees from Allergan Inc. and Abbvie Pharmaceuticals and has been on the advisory boards of Allergan Inc, Shire and Salix Pharmaceuticals.

\section{References}

1. Probiotic market by application (food and beverages, dietary supplements, animal feed) by end users (human probiotics, animal probiotics)-global industry analysis, size, share, growth and forecast 2014-2020. Albany (NY): Transparency Market Research; 2015. Available: www.transparencymarketresearch.com/probiotics-market.html (accessed 2017 Jan. 6).

2. Dinan TG, Cryan JF. Gut-brain axis in 2016: brain-gut-microbiota axis - mood, metabolism and behaviour. Nat Rev Gastroenterol Hepatol 2017;14:69-70.

3. Foster JA. Gut microbiome and behavior: focus on neuroimmune interactions. Int Rev Neurobiol 2016;131:49-65.

4. Anglin R, Surette M, Moayyedi P, et al. Lost in translation: the gut microbiota in psychiatric illness. Can J Psychiatry 2015;60:460-3.

5. Bercik P, Denou E, Collins J, et al. The intestinal microbiota affect central levels of brain-derived neurotrophic factor and behavior in mice. Gastroenterology 2011;141:599-609.

6. Bravo JA, Forsythe P, Chew MV, et al. Ingestion of Lactobacillus strain regulates emotional behavior and central GABA receptor expression in a mouse via the vagus nerve. Proc Natl Acad Sci U S A 2011;108: 16050-5.
7. Bercik P, Park AJ, Sinclair D, et al. The anxiolytic effect of Bifidobacterium longum NCC3001 involves vagal pathways for gutbrain communication. Neurogastroenterol Motil 2011;23:1132-9.

8. Lyte M, Li W, Opitz N, et al. Induction of anxiety-like behavior in mice during the initial stages of infection with the agent of murine colonic hyperplasia Citrobacter rodentium. Physiol Behav 2006; 89:350-7

9. Goehler LE, Gaykema RP, Opitz N, et al. Activation in vagal afferents and central autonomic pathways: early responses to intestinal infection with Campylobacter jejuni. Brain Behav Immun 2005;19:334-44.

10. Bharwani A, Mian MF, Foster JA, et al. Structural \& functional consequences of chronic psychosocial stress on the microbiome \& host. Psychoneuroendocrinology 2016;63:217-27.

11. Bailey MT, Dowd SE, Galley JD, et al. Exposure to a social stressor alters the structure of the intestinal microbiota: implications for stressorinduced immunomodulation. Brain Behav Immun 2011;25:397-407.

12. Li W, Dowd SE, Scurlock B, et al. Memory and learning behavior in mice is temporally associated with diet-induced alterations in gut bacteria. Physiol Behav 2009;96:557-67.

13. Ford AC, Quigley EM, Lacy BE, et al. Efficacy of prebiotics, probiotics, and synbiotics in irritable bowel syndrome and chronic idiopathic constipation: systematic review and meta-analysis. Am J Gastroenterol 2014;109:1547-61.

14. Steenbergen L, Sellaro R, van Hemert S, et al. A randomized controlled trial to test the effect of multispecies probiotics on cognitive reactivity to sad mood. Brain Behav Immun 2015;48:258-64.

15. Schmidt K, Cowen PJ, Harmer CJ, et al. Prebiotic intake reduces the waking cortisol response and alters emotional bias in healthy volunteers. Psychopharmacology (Berl) 2015;232:1793-801.

16. Tillisch K, Labus J, Kilpatrick L, et al. Consumption of fermented milk product with probiotic modulates brain activity. Gastroenterology 2013;144:1394-401.

17. Jiang $\mathrm{H}$, Ling Z, Zhang $\mathrm{Y}$, et al. Altered fecal microbiota composition in patients with major depressive disorder. Brain Behav Immun 2015;48:186-94

18. Zheng P, Zeng B, Zhou C, et al. Gut microbiome remodeling induces depressive-like behaviors through a pathway mediated by the host's metabolism. Mol Psychiatry 2016;21:786-96.

19. Naseribafrouei A, Hestad K, Avershina E, et al. Correlation between the human fecal microbiota and depression. Neurogastroenterol Motil 2014;26:1155-62.

20. Kelly JR, Borre Y, O'Brien C, et al. Transferring the blues: depression-associated gut microbiota induces neurobehavioural changes in the rat. J Psychiatr Res 2016;82:109-18.

21. Aizawa E, Tsuji H, Asahara T, et al. Possible association of Bifidobacterium and Lactobacillus in the gut microbiota of patients with major depressive disorder. J Affect Disord 2016;202:254-7.

22. Tomasik J, Yolken RH, Bahn S, et al. Immunomodulatory effects of probiotic supplementation in schizophrenia patients: a randomized, placebo-controlled trial. Biomark Insights 2015;10:47-54

23. Akkasheh G, Kashani-Poor Z, Tajabadi-Ebrahimi M, et al. Clinical and metabolic response to probiotic administration in patients with major depressive disorder: a randomized, double-blind, placebo-controlled trial. Nutrition 2016;32:315-20.

24. Dinan TG, Cryan JF. Mood by microbe: towards clinical translation. Genome Med 2016;8:36.

25. Marshall JK, Thabane M, Garg AX, et al. Eight year prognosis of postinfectious irritable bowel syndrome following waterborne bacterial dysentery. Gut 2010;59:605-11.

26. Jones R, Latinovic R, Charlton J, et al. Physical and psychological comorbidity in irritable bowel syndrome: a matched cohort study using the General Practice Research Database. Aliment Pharmacol Ther 2006;24:879-86.

27. Ananthakrishnan AN, Gainer VS, Cai T, et al. Similar risk of depression and anxiety following surgery or hospitalization for Crohn's disease and ulcerative colitis. Am J Gastroenterol 2013;108:594-601.

28. Ford AC, Bercik P, Morgan DG, et al. Characteristics of functional bowel disorder patients: a cross-sectional survey using the Rome III criteria. Aliment Pharmacol Ther 2014;39:312-21.

29. Roy-Byrne PP, Davidson KW, Kessler RC, et al. Anxiety disorders and comorbid medical illness. Gen Hosp Psych 2008;30:208-25.

30. Liu Y, Zhang L, Wang X, et al. Similar fecal microbiota signatures in patients with diarrhea-predominant irritable bowel syndrome and patients with depression. Clin Gastroenterol Hepatol 2016;14:1602-61. 